\title{
Mitochondrial And Myogenic Gene Expression Pathways Are Influenced By The Exercise Mimetic Formoterol In Human Myotubes
}

Anthony A. Duplanty ( $\sim$ aduplanty@twu.edu )

Texas Woman's University

Emily L. Zumbro

Texas Woman's University

Ryan A. Gordon

Texas Woman's University

Gena D. Guerin

Texas Woman's University

Matthew F. Brisebois

Texas Woman's University

Matthew L. Sokoloski

Texas Woman's University

Brandon R. Rigby

Texas Woman's University

\section{Research Article}

Keywords: skeletal muscle, myoblast, myotube, myogenesis, mitochondria, thyroid, exercise mimetic

Posted Date: December 8th, 2021

DOI: https://doi.org/10.21203/rs.3.rs-1136059/v1

License: (c) (i) This work is licensed under a Creative Commons Attribution 4.0 International License. Read Full License 


\section{Abstract}

Background: Exercise is an effective treatment for establishing and maintaining skeletal muscle (SKM) health. The interconnected cascade of gene expression pathways related to myogenesis, mitochondrial homeostasis, and thyroid hormone metabolism are critical to SKM health. This in vitro study was conducted to investigate the effects of exercise mimetic (formoterol) stimulation on human SKM cell signaling during myogenesis, and to provide insight on potential targets for future studies exploring therapies for SKM atrophy.

Methods: Human myoblasts were cultured and differentiated to evaluate the effects of exercise mimetic stimulation on gene expression during mid and late myogenesis. We characterized the expression of 24 genes related to myogenesis, mitochondrial biogenesis, thyroid hormone metabolism, and cellular homeostasis.

Results: Formoterol stimulated the gene expression for SKM pathways related to mitochondrial biogenesis, thyroid metabolism, and cellular homeostasis. Additionally, formoterol resulted in a myogenic program that appears to favor prolonged myoblast proliferation and delayed myotube maturation.

Conclusion: Robust, yet differential effects of exercise mimetic stimulation on gene expression during mid-myogenesis and at terminal differentiation were found. The results of our study support the groundwork for establishing further experiments utilizing exercise signaling as a therapeutic treatment in models targeting dysfunctional SKM cell growth.

\section{Background}

The benefits of cultivating and maintaining healthy skeletal muscle (SKM) throughout life are numerous and potent [1-4]. Loss of muscle mass, whether initiated by disease (atrophy) or by decades of sedentary lifestyle (sarcopenia), often results in decreased quality of life and increased morbidity $[5,6]$. Exercise, especially modes that induce SKM hypertrophy, has been established as an effective treatment for attenuating or reversing SKM loss [5, 7]. However, the interconnected scope of effects that exercise signaling has on multiple molecular pathways within SKM cells remains largely uncharacterized. Thus, there is importance in further elucidating the exercise-stimulated response of molecules involved in the many physiological processes of human SKM growth, regeneration, repair, reactive oxidative species (ROS) mitigation, hormonal signal transduction, as well as factors related to atrophy.

Myogenesis, a process of SKM regeneration and repair, is the control of genetic networks that activate satellite cells (i.e. myoblasts) and initiate their differentiation into mature myofibers [8-10]. This process is regulated by the expression of myogenic regulatory factors (MRFs) such as MYF5, MYOD, MYOF, and MEF2c, which coordinate the process of myoblast proliferation and myotube differentiation. These factors regulate tissue repair and regeneration that is influenced by both disease and exercise. Additionally, SKM can further grow when protein synthesis is upregulated by mechanistic target of 
rapamycin (mTOR) signaling, a protein kinase that regulates protein synthesis especially in response to exercise-induced mechanical stimulation [11-13].

Within the SKM cell, in addition to providing the foundation for healthy mitochondrial function, the maintenance of mitochondrial homeostasis is essential for promoting balanced myofibrillar protein synthesis, new growth, and regeneration $[5,14,15]$. Further, the dysfunction of mitochondrial processes (e.g. biogenesis, respiration, mitigation of oxidative stress) within the SKM cell contributes to the etiology of sarcopenia $[5,16,17]$. Several important transcription factors contribute to SKM mitochondrial homeostasis and were investigated in this study. Peroxisome proliferator-activated receptor gamma coactivator 1-alpha/beta (PGC-1a and PGC-1 $\beta$ ), mitochondrial transcription factor A (TFAM), and nuclear respiratory factor $1 / 2$ (NRF1, NRF2) are regulators of mitochondrial homeostasis and promoters of mitochondrial biogenesis [18]. As mitochondrial health is related to processes involved in overall cellular and organismal health, both directly and indirectly, it is therefore an important therapeutic target for mitigating SKM dysfunction and preventing sarcopenia $[5,19]$.

Another important, albeit less studied factor for SKM health is the metabolism of thyroid hormone (TH) within the SKM cell. Once transported into the SKM cell, deiodinases 2 and 3 (DIO2 and DIO3 respectively) convert TH to the active form, triiodothyronine (T3) or the inactive form, reverse T3 (rT3) [20]. Active T3 stimulates specific TH nuclear receptors (e.g. THRa) aiding in the regulation of cellular metabolism, myogenesis, mitochondrial homeostasis, and calcium homeostasis [20]. Thus, the inclusion of TH metabolism in the present study is of importance to highlight the significance of TH action on SKM processes, particularly when an exercise mimetic is introduced into the SKM cell.

Exercise mimetic stimulation of SKM, specifically the $\beta 2$-adrenergic receptor (B2AR) pathway via formoterol treatment, has been used to increase mitochondrial biogenesis [21-23], oxidative metabolism [24], and influence the modulation of myoblast differentiation [25]. Individually, many genes related to SKM processes have been investigated using exercise-mimetic stimulation designs, usually in reference to a limited range of pathways. To date, genes investigated using these designs have been mostly studied using animal models. However, gene expression does differ between rodents, humans, and nonhuman primates, thereby making translations to human physiology based on animal model data confounding. For example, in humans, TH stimulation of PGC-1a occurs indirectly via T3 stimulation of AMPK, but happens directly by T3 in rodents [26]. Thus, characterization of multiple gene expression pathways relating to exercise-stimulation in human SKM is warranted.

SKM exhibits high levels of plasticity and is influenced by a wide range of epigenetic stimuli. Crosssectional analysis of SKM tissue signaling in vivo reveals various stages of ongoing myogenesis, thus limiting conclusions made at one discrete time point. Therefore, we developed a purpose-designed in vitro culture model to isolate SKM cells (during mid and late myogenesis) and investigate the influence of exercise-related signaling (via formoterol stimulation) on the expression of 24 genes related to myogenesis, mitochondrial biogenesis, TH metabolism, and cellular homeostasis. We hypothesized that the results of our study will deliver insights on the inner workings of a complex network of gene 
expression programs and inform on future experiments that can utilize similar methods for investigating the effects of exercise-related signaling on muscle growth and atrophy in SKM disease.

\section{Methods}

\section{Cell culture and myotube formation}

Primary human SKM myoblasts obtained from healthy adult donors (Sigma-Aldrich, St. Louis, MO, USA) were cultured in $35 \mathrm{~mm}$ 6-well collagen coated plates (Gibco, New York, NY, USA) at $37^{\circ} \mathrm{C}$ in $5 \% \mathrm{CO}_{2}$ in growth media (151-500 Sigma-Aldrich). Myoblasts were seeded at a density of 80,000 cells per well and once they reached 80-90\% confluency, differentiation medium (151D-250 Sigma-Aldrich) was used to initiate myotube formation and replaced every 48 hours.

\section{Exercise mimetic stimulation}

Formoterol fumarate dehydrate $>98 \%$ HPLC (F9552 Sigma-Aldrich), was reconstituted in dimethyl sulfoxide (DMSO) and mixed into differentiation medium at a concentration of 30nM. DMSO was used as a control by adding a volume equal to formoterol to the differentiation media for all CON groups. Four 6-well plates were concomitantly cultured and represent the following groups: day 4 (mid-myogenesis) with/without formoterol treatment (D4 CON and D4 FORM respectively) and day 6 (mature myotube formation) with/without formoterol treatment (D6 CON and D6 FORM respectively). Three hours before scheduled cell extraction (day 4 and day 6), differentiation media was changed to either fresh control media or formoterol-treated media for the duration of the incubation period.

\section{Gene expression}

Cells were extracted at days 4 (mid-myogenesis) and 6 (mature myotubes) of myogenesis. Total RNA was extracted using a Qiagen miRNeasy kit (Qiagen, Germantown, MD, USA). Complementary DNA was synthesized from $1 \mu \mathrm{g}$ of the resulting total RNA using the High Capacity cDNA Reverse Transcription Kit (Applied Biosystems, Carlsbad, CA, USA). Real-time quantitative polymerase chain reaction (qPCR) detection was performed in duplicate using PowerUp SYBR and the QuantStudio RealTime 3 PCR System (Applied Biosystems, Carlsbad, CA, USA). Forward and reverse primers from IDT (Integrated DNA Technologies, Coralville, IA, USA) were used and are listed in Table 1. Data were analyzed using the comparative $\mathrm{Ct}(\Delta \Delta \mathrm{Ct})$ method for quantification. Ribosomal protein S13 (RPS13) was used as the endogenous control for comparative data of target genes and the experimental group data was normalized to D4 CON values.

\section{Statistical analyses}

Data are presented as mean \pm standard error of the mean (SEM) and statistical significance was set a $P<$ 0.05. Statistical significance of the differences between the means was evaluated using one-way 
analysis of variance test (ANOVA), with Tukey's HSD post-test. Statistical analysis was performed using IBM SPSS Statistics for Windows, Version 25.0 (IBM Corp, Armonk, NY, USA).

\section{Results}

\section{Formoterol influences SKM gene expression related to myogenesis}

In this study, we used a model of investigating gene expression during myogenesis in human myoblasts to provide insight into the effects of this process in response to exercise-mimetic stimulation in vitro. MYF5, a promoter of myoblast proliferation [9], was increased for D6 FORM compared to D4 FORM, D4 CON, and D6 CON. D6 CON was significantly lower than D4 CON (Fig. 1a). MYOD influences the commitment of myoblasts into the process of differentiating into mature myotubes and MYOG further directs the maturing differentiating process [9]. We found similar results for both MYOD and MYOG as expression was lower for D4 FORM compared to D4 CON. Additionally, for both day 6 groups MYOD and MYOG was reduced compared to D4 CON, and D6 FORM was lower compared to D6 CON (Fig. 1b and 1c). MEF2c, a potentiator of the transcriptional effects of the MYFs, was decreased for D6 FORM compared to D6 CON, further indicating less commitment to the maturing of myotubes (Fig. 1d). Further, PGC-1a4 expression was significantly greater for D6 FORM compared to D4 and D6 CON (Fig. 1e). The expression of mTOR was significantly decreased for D6 CON compared to D4 CON, however, this day 6 decrease in mTOR was notably absent in the D6 FORM group (Fig. 1f).

\section{Formoterol stimulates expression of genes related to SKM mitochondrial biogenesis}

Healthy mitochondrial function is essential for muscle growth, especially in response to energetic or oxidative demand (e.g. exercise) [5,27]. We analyzed several genes related to the production of essential proteins for mitochondrial biogenesis. Formoterol treatment appeared to upregulate mitochondrial biogenic activity overall during myogenesis, similar to exercise stimulation. PGC-1 a was increased from D4 CON to D6 CON (Fig. 2a). Formoterol treatment caused a robust day 4 and 6 increase of PGC-1a compared to D4 CON (Fig. 2a). PGC-1 $\beta$ was increased for both day 4 and day 6 FORM groups compared to D4 CON, and D6 FORM was significantly greater than D6 CON (Fig. 2b). For TFAM, D6 FORM was greater than both D4 and D6 CON groups (Fig. 2C). NRF1 was decreased for all groups compared to D4 CON (Fig. 2d). NRF2 increased at D6 CON and D6 FORM compared to D4 CON, however, D6 FORM was found to be significantly lower than D6 CON (Fig. 2e). Superoxide dismutase 2 (SOD2), the mitochondrial specific antioxidant, was increased for D6 CON compared to D4 CON, and D6 FORM was found to be significantly lower than D6 CON, indicating a lesser presence of the superoxide anion at D6 with formoterol stimulation (Fig. 2f).

\section{Formoterol affects intracellular SKM activity of genes related to thyroid hormone metabolism}

Formoterol stimulation resulted in the reduced expression of THRa for both day 4 and day 6 myogenesis compared to D4 CON (Fig. 3a). Despite a decreased signal for THRa expression, DIO2 expression was increased by formoterol treatment for day 4 and day 6 as compared to D4 CON, with D6 FORM also being 
significantly greater than D6 CON (Fig. 3b). DIO3 was greater at D4 FORM and D6 FORM compared to D4 CON and D6 CON, respectively (Fig. 3c). Within SKM, intracellular calcium is managed by sarco/endoplasmic reticulum $\mathrm{Ca}^{2+}{ }^{-}$ATPase 1 and 2 (SERCA1/2) which, in turn, is upregulated by T3 and exercise [28]. SERCA 1 and 2 expression remained unchanged by formoterol stimulation, SERCA1 in D6 CON was decreased compared to D4 CON (Fig. 3d and 3e). Expression of ERRa was increased for D6 CON compared to D4 CON. Additionally, D6 FORM was significantly lower compared to D6 CON, but not different from D4 CON (Fig. 3f).

\section{Formoterol increases SKM $\beta 2$-adrenergic receptor gene expression and influences genes related to cellular homeostasis}

To identify the effects of exercise stimulation on cellular homeostasis during SKM myogenesis, several genes related to bioenergetics, cell atrophy, ROS mitigation, as well as B2AR were targeted. We found that formoterol stimulation increased B2AR expression at day 6 of myogenesis (Fig. 4a). AMPK and the autophagy-related marker (ATG5) [29] were unchanged for all groups (Fig. $4 \mathrm{~b}$ and 4c). The glucose transporter, (GLUT4) [30] was increased for both D4 FORM and D6 FORM compared to D4 CON and D6 CON respectively (Fig. 4d). Glutathione synthetase (GSS), a precursor to glutathione, was decreased for all treatment groups compared to D4 CON (Fig. 4e). Also related to autophagy, forkhead box (FOXO3), was decreased for the CON group at D6 (Fig. 4f).

\section{Discussion}

In this study, we utilized an in vitro SKM cell culture model to investigate the influence of exercise-related signaling on the expression of 24 genes related to myogenesis, mitochondrial biogenesis, thyroid hormone metabolism, and cellular homeostasis. Our study is novel in that multiple SKM gene expression pathways were concomitantly analyzed and interpreted together, not only for terminal stage myotubes, but also during mid-myogenesis.

Skeletal muscle cells naturally cycle through periods of both protein synthesis and protein breakdown, all of which occurs during the various stages of myogenesis throughout life. Imbalances to these processes result in increased proteolysis, dysregulated mitigation of ROS, mitochondrial dysfunction, and eventual loss of SKM mass and function [6, 7]. Exercise has many beneficial effects on muscle health and is often prescribed as a treatment for preventing or attenuating sarcopenia and pathological atrophy. However, the cross-pathway mapping of exercise-stimulated gene expression has not been fully elucidated, especially in human tissue.

Formoterol is a long-acting $\beta 2$-adrenergic receptor agonist that has been effectively used as an exercise mimetic with in vivo and in vitro animal models, stimulating many pathways within SKM tissue [21, 22, 31]. Specifically, in rodent models, formoterol increases PGC-1a, mitochondrial respiration, morphology, and biogenesis [21-23]. In rhesus macaques, formoterol treatment increases PGC-1a expression, mitochondrial DNA quantity, and improves mitochondrial respiration of unhealthy myoblasts [31]. 
Stimulation of B2AR signaling has been implicated as a potential therapeutic target for skeletal muscle wasting disorders, due to this pathway's influence on regulating SKM protein synthesis and degradation [32-33]. While the use of pharmaceutical therapy utilizing B2AR agonists is currently limited for people experiencing muscular atrophy or disease, it is clear that the B2AR pathway plays a significant role in the regulation of SKM growth [34], especially in the context of exercise signaling. Additionally, the B2AR pathway is an upstream signaling cascade that is activated by both endurance and resistance exercise and stimulated by epinephrine and norepinephrine. This makes the pathway ideal for in vitro exercise stimulation, rather than stimulating individual downstream molecules with mimetics such as AICAR, which only affects APMK [35].

The mechanisms of myogenic regulation have been well characterized $[9,10]$. However, this regulation has not been well studied in human myotubes throughout the various stages of myogenesis, especially in the context of exercise stimulation. In this study, our exercise mimetic model induced a myogenic program that appears to prolong myoblast proliferation and delay maturation of myotubes, as evidenced by the changes in MYF5, MYOD, and MYOG for both D4 FORM and D6 FORM. The influence of formoterol on the MRFs in this study is unique, as increases in MYOD and MYOG gene expression following exercise have been previously reported [37]. Based on these results, the effects of exercise signaling on myogenesis may be influenced by the timing of the stimulus in relation to what stage the myotubes are on within the myogenic program (i.e. mid- or late-stage myogenesis). Thus, exercise stimuli could interfere with the regeneration or repair of SKM tissue by delaying or prolonging myogenesis. This information may be meaningful in the context of SKM growth and regeneration, as it indicates the timing of additional exercise stimuli should be strategically considered due to its ability to influence the timeline of myotube formation and maturity. To investigate muscle growth during myogenesis, we analyzed the gene expression for two important factors that regulate protein synthesis in response to exercise signaling, including PGC-1a4 [an isoform of PGC-1a, which is a promoter of resistance exercise-induced hypertrophy [37] and the protein kinase mTOR. We found that formoterol treatment may allow an increase in protein synthesis, despite delayed signaling for myotube formation. These results could indicate greater cellular energetic demands directed towards myotube formation and differentiation in the CON groups, whereas formoterol may have triggered processes more geared towards protein synthesis and myoblast proliferation.

Autophagy is a normal cellular process that removes damaged organelles while recycling functional cellular components that fuse with proximal healthy cells and is an important process during cell differentiation. The regulation of autophagy in healthy cells aims to optimize tissue function as a whole and prevent the accumulation of dysfunctional cells and cellular components, performing as a quality control mechanism. ATG5, a regulator of autophagy, is typically upregulated throughout the myogenic process and is responsible for myotube fusion [38]. Alternatively, exercise upregulates ATG5 in vivo [29], implicating autophagy to be a primary contributing mechanism of myogenesis and tissue repair. Regulation of the autophagy system declines linearly with age [39], but is augmented by chronic exercise training, highlighting the importance of regular exercise in regulating cellular processes throughout the lifespan [40]. Interestingly, we found no changes in ATG5 between conditions in this study. However, we 
found differential responses for ROS mitigation. Skeletal muscle is a major site of ROS generation as a result of energy production and muscle contraction. The accumulation of ROS is detrimental to organismal and tissue health and function with profound tissue dysregulation and inflammation evident as a result [41]. Efficient mechanisms are in place to minimize the accumulation of ROS and prevent damage to DNA, as found in aging muscle [41]. We found reduced GSS expression for all groups compared to D4 CON, and elevated SOD2, a mitochondrial related antioxidant enzyme, for both D6 groups.

The peripheral effects of thyroid hormone signaling within SKM are substantial and play crucial roles in metabolism, function, and growth [42]. Analyzing the expression of THRa and the deiodinases DIO2 and DIO3 is of particular interest in helping to characterize SKM homeostasis due to their regulatory roles in mitochondrial biogenesis and myogenesis [43]. Our results indicate robust activity of TH metabolism in response to exercise mimetic stimulation within SKM. In particular, the increases in DIO2 found in the FORM groups, which indicate increased activation of T4 into T3 within SKM, lead to stimulation of nuclear targets specific to metabolism and myogenesis [20]. To further investigate TH metabolism in connection to mitochondrial homeostasis, we analyzed estrogen-related receptor-a (ERRa), a nuclear receptor that works in tangent with $\mathrm{TH}$ receptors, and is co-activated by PGC-1a to regulate mitochondrial processes such as biogenesis, mitophagy, fission, and oxidative phosphorylation. Interestingly, ERRa was elevated at D6 CON but not D6 FORM, despite significant increases in PGC-1a for both groups. We can therefore hypothesize that mitochondrial biogenesis still occurred in the absence of ERRa stimulation, as increases for both PGC-1 $a$ and TFAM in the D6 FORM group, were also observed. One explanation for elevated ERRa in the D6 CON group may be related to the increased NRF2 and SOD2 expression found for this condition. This likely indicates an increased need for oxidative phosphorylation and mitigation of ROS, which may have resulted from less mitochondrial biogenesis than the FORM group.

\section{Conclusion}

Formoterol stimulated the gene expression for SKM pathways related to mitochondrial biogenesis, thyroid metabolism, and cellular homeostasis. Additionally, formoterol resulted in a myogenic program that appears to favor prolonged myoblast proliferation and delayed myotube maturation. The results in this study provide the groundwork for mechanistic studies that require controlling for the vast array of effects that external stimuli (e.g. age, pathology, nutrition, medication) can have on the wide range of physiological processes in human SKM. We simultaneously examined the overlapping and interconnected functions of the many different and complex intracellular components that regulate SKM growth and physiology, which should be a key feature of future research in this topic. Current and future research is being aimed at discovering the specific exercise strategies, nutritional interventions, and medical practices that will most effectively support the new growth of SKM for those who are experiencing progressive loss of skeletal muscle mass. In many cases, sarcopenia and atrophy that is pathologic in origin (e.g. alcoholic myopathy, disuse atrophy, and cancer cachexia) may be treated, in significant part, by exercise prescription. 


\section{Abbreviations}

(AMPK): AMP-activated protein kinase; (ANOVA): Analysis of variance; (ATG5): Autophagy related 5; (B2AR): 32 -adrenergic receptor; (DIO2): Deiodinase 2; (DIO3): Deiodinase 3; (DMSO): Dimethyl sulfoxide; (ERRa): Estrogen related receptor alpha; (FOXO3): Forkhead box 03; (GLUT4): Glucose transporter 4; (GSS): Glutathione synthetase; (MEF2c): Myocyte enhancer factor 2; (MRFs): Myogenic regulatory factors; (mTOR): Mechanistic target of rapamycin; (MYF5): Myogenic factor 5; (MYOD): Myogenic differentiation 1; (MYOG): Myogenin; (NRF1): Nuclear respiratory factor 1; (NRF2): Nuclear respiratory factor 2; (PGC-1a): Peroxisome proliferator-activated receptor gamma coactivator 1-alpha; (PGC-1a4): PGC-1a isoform 4; (PGC-1 $\beta$ ): Peroxisome proliferator-activated receptor gamma coactivator 1-beta; (qPCR): Quantitative polymerase chain reaction; (ROS): Reactive oxidative species; (RPS13): Ribosomal protein S13; (rT3): Reverse T3; (SEM): Standard error of the mean; (SERCA1): Sarco/endoplasmic reticulum Ca2+ ATPase isoform 1; (SERCA1): Sarco/endoplasmic reticulum Ca2+ ATPase isoform 2; (SKM): Skeletal muscle; (SOD2): Superoxide dismutase 2; (T3): Triiodothyronine; (TFAM): Mitochondrial transcription factor $\mathrm{A} ;(\mathrm{TH})$ : Thyroid hormone; (THR): Thyroid hormone receptor alpha

\section{Declarations}

\section{Ethics approval and consent to participate}

All experimental procedures using human tissue were approved by the Texas Woman's University Institutional Review Board (IRB-FY2021-71).

\section{Consent for publication}

Not applicable

\section{Availability of data and materials}

The datasets used and analyzed during the current study are available from the corresponding author on reasonable request.

\section{Competing interests}

The authors declare that they have no competing interests.

\section{Funding}

We acknowledge the funding support from a Texas Woman's University Research Enhancement Program Award (A.D.). Additionally, research reported in this publication was supported in part by the National Institutes of Health under award number T32 HL007457 (E.Z.). The content is solely the responsibility of the authors and does not necessarily represent the official views of the National Institutes of Health.

\section{Authors' contributions}


A.D designed and managed the study. A.D., E.Z, R.G. G.G., M.B., M.S., and B.R performed experiments, analyzed the data, and wrote the manuscript. All authors read and approved the final manuscript.

\section{Acknowledgements}

We gratefully acknowledge Dr. George King, Director, School of Health Promotion and Kinesiology at Texas Woman's University for support of our project. We are also grateful to Dr. Vic Ben-Ezra for his mentorship and review of the manuscript.

\section{References}

1. Watson K, Baar K. mTOR and the health benefits of exercise. Semin Cell Dev Biol. 2014 Dec;36:130-9. doi: 10.1016/j.semcdb.2014.08.013.

2. Galloza J, Castillo B, Micheo W. Benefits of Exercise in the Older Population. Phys Med Rehabil Clin N Am. 2017 Nov;28(4):659-669. doi: 10.1016/j.pmr.2017.06.001.

3. Piercy KL, Troiano RP, Ballard RM, Carlson SA, Fulton JE, Galuska DA, George SM, Olson RD. The Physical Activity Guidelines for Americans. JAMA. 2018 Nov 20;320(19):2020-2028. doi: 10.1001/jama.2018.14854.

4. Nieman DC, Pence BD. Exercise immunology: Future directions. J Sport Health Sci. 2020 Sep;9(5):432-445. doi: 10.1016/j.jshs.2019.12.003.

5. Carter HN, Chen CC, Hood DA. Mitochondria, muscle health, and exercise with advancing age. Physiology (Bethesda). 2015 May;30(3):208-23. doi: 10.1152/physiol.00039.2014.

6. Wilkinson DJ, Piasecki M, Atherton PJ. The age-related loss of skeletal muscle mass and function: Measurement and physiology of muscle fibre atrophy and muscle fibre loss in humans. Ageing Res Rev. 2018 Nov;47:123-132. doi: 10.1016/j.arr.2018.07.005.

7. Giallauria F, Cittadini A, Smart NA, Vigorito C. Resistance training and sarcopenia. Monaldi Arch Chest Dis. 2016 Jun 22;84(1-2):738. doi: 10.4081/monaldi.2015.738.

8. Le Grand F, Rudnicki M. Satellite and stem cells in muscle growth and repair. Development. 2007 Nov;134(22):3953-7. doi: 10.1242/dev.005934.

9. Bentzinger CF, Wang YX, Rudnicki MA. Building muscle: molecular regulation of myogenesis. Cold Spring Harb Perspect Biol. 2012 Feb 1;4(2):a008342. doi: 10.1101/cshperspect.a008342.

10. Chal J, Pourquié O. Making muscle: skeletal myogenesis in vivo and in vitro. Development. 2017 Jun 15;144(12):2104-2122. doi: 10.1242/dev.151035.

11. Bodine SC. mTOR signaling and the molecular adaptation to resistance exercise. Med Sci Sports Exerc. 2006 Nov;38(11):1950-7. doi: 10.1249/01.mss.0000233797.24035.35.

12. Yoon MS. mTOR as a key regulator in maintaining skeletal muscle mass. Front Physiol. 2017;8:788. https://doi.org/10.3389/fphys.2017.00788.

13. Duplanty AA, Budnar RG, Luk HY, Levitt DE, Hill DW, McFarlin BK, Huggett DB, Vingren JL. Effect of Acute Alcohol Ingestion on Resistance Exercise-Induced mTORC1 Signaling in Human Muscle. J 
Strength Cond Res. 2017 Jan;31(1):54-61. doi: 10.1519/JSC.0000000000001468.

14. Wagatsuma A, Sakuma K. Mitochondria as a potential regulator of myogenesis. ScientificWorldJournal. 2013;2013:593267. doi: 10.1155/2013/593267.

15. Romanello V, Sandri M. Mitochondrial Quality Control and Muscle Mass Maintenance. Front Physiol. 2016 Jan 12;6:422. doi: 10.3389/fphys.2015.00422.

16. Marzetti E, Calvani R, Cesari M, Buford TW, Lorenzi M, Behnke BJ, Leeuwenburgh C. Mitochondrial dysfunction and sarcopenia of aging: from signaling pathways to clinical trials. Int J Biochem Cell Biol. 2013 Oct;45(10):2288-301. doi: 10.1016/j.biocel.2013.06.024.

17. Hepple RT. Mitochondrial involvement and impact in aging skeletal muscle. Front Aging Neurosci. 2014 Sep 10;6:211. doi: 10.3389/fnagi.2014.00211.

18. Yin F, Cadenas E. Mitochondria: the cellular hub of the dynamic coordinated network. Antioxid Redox Signal. 2015 Apr 20;22(12):961-4. doi: 10.1089/ars.2015.6313.

19. Safdar A, Hamadeh MJ, Kaczor JJ, Raha S, Debeer J, Tarnopolsky MA. Aberrant mitochondrial homeostasis in the skeletal muscle of sedentary older adults. PLoS One. 2010 May 24;5(5):e10778. doi: 10.1371/journal.pone.0010778.

20. Lee JW, Kim NH, Milanesi A. Thyroid Hormone Signaling in Muscle Development, Repair and Metabolism. J Endocrinol Diabetes Obes. 2014 Jul-Sep;2(3):1046.

21. Miura S, Kawanaka K, Kai Y, Tamura M, Goto M, Shiuchi T, Minokoshi Y, Ezaki O. An increase in murine skeletal muscle peroxisome proliferator-activated receptor-gamma coactivator-1alpha (PGC1alpha) mRNA in response to exercise is mediated by beta-adrenergic receptor activation. Endocrinology. 2007 Jul;148(7):3441-8. doi: 10.1210/en.2006-1646.

22. Wills LP, Trager RE, Beeson GC, Lindsey CC, Peterson YK, Beeson CC, Schnellmann RG. The $\beta 2-$ adrenoceptor agonist formoterol stimulates mitochondrial biogenesis. J Pharmacol Exp Ther. 2012 Jul;342(1):106-18. doi: 10.1124/jpet.112.191528.

23. Azevedo Voltarelli V, Coronado M, Gonçalves Fernandes L, Cruz Campos J, Jannig PR, Batista Ferreira JC, Fajardo G, Chakur Brum P, Bernstein D. $\beta_{2}$-Adrenergic Signaling Modulates Mitochondrial Function and Morphology in Skeletal Muscle in Response to Aerobic Exercise. Cells. 2021 Jan 13;10(1):146. doi: 10.3390/cells10010146.

24. Pearen MA, Myers SA, Raichur S, Ryall JG, Lynch GS, Muscat GE. The orphan nuclear receptor, NOR-1, a target of beta-adrenergic signaling, regulates gene expression that controls oxidative metabolism in skeletal muscle. Endocrinology. 2008 Jun;149(6):2853-65. doi: 10.1210/en.2007-1202.

25. Kim SH, Yi SJ, Lee H, Kim JH, Oh MJ, Song EJ, Kim K, Jhun BH. $\beta_{2}$-Adrenergic receptor $\left(\beta_{2}\right.$-AR) agonist formoterol suppresses differentiation of L6 myogenic cells by blocking PI3K-AKT pathway. Anim Cells Syst (Seoul). 2019 Feb 22;23(1):18-25. doi: 10.1080/19768354.2018.1561516.

26. Barbe P, Larrouy D, Boulanger C, Chevillotte E, Viguerie N, Thalamas C, Oliva Trastoy M, Roques M, Vidal H, Langin D. Triiodothyronine-mediated up-regulation of UCP2 and UCP3 mRNA expression in human skeletal muscle without coordinated induction of mitochondrial respiratory chain genes. FASEB J. 2001 Jan;15(1):13-15. doi: 10.1096/fj.00-0502fje. 
27. Ferri E, Marzetti E, Calvani R, Picca A, Cesari M, Arosio B. Role of Age-Related Mitochondrial Dysfunction in Sarcopenia. Int J Mol Sci. 2020 Jul 23;21(15):5236. doi: 10.3390/ijms21155236.

28. Kubo H, Libonati JR, Kendrick ZV, Paolone A, Gaughan JP, Houser SR. Differential effects of exercise training on skeletal muscle SERCA gene expression. Med Sci Sports Exerc. 2003 Jan;35(1):27-31. doi: 10.1097/00005768-200301000-00005.

29. Rocchi A, He C. Regulation of Exercise-Induced Autophagy in Skeletal Muscle. Curr Pathobiol Rep. 2017;5(2):177-186. doi:10.1007/s40139-017-0135-9

30. Richter EA, Hargreaves M. Exercise, GLUT4, and skeletal muscle glucose uptake. Physiol Rev. 2013 Jul;93(3):993-1017. doi: 10.1152/physrev.00038.2012.

31. Duplanty AA, Siggins RW, Allerton T, Simon L, Molina PE. Myoblast mitochondrial respiration is decreased in chronic binge alcohol administered simian immunodeficiency virus-infected antiretroviral-treated rhesus macaques. Physiol Rep. 2018 Mar;6(5):e13625. doi: 10.14814/phy2.13625.

32. Koopman R, Ryall JG, Church JE, Lynch GS. The role of beta-adrenoceptor signaling in skeletal muscle: therapeutic implications for muscle wasting disorders. Curr Opin Clin Nutr Metab Care. 2009 Nov;12(6):601-6. doi: 10.1097/MC0.0b013e3283318a25.

33. Joassard OR, Amirouche A, Gallot YS, Desgeorges MM, Castells J, Durieux AC, Berthon P, Freyssenet DG. Regulation of Akt-mTOR, ubiquitin-proteasome and autophagy-lysosome pathways in response to formoterol administration in rat skeletal muscle. Int J Biochem Cell Biol. 2013 Nov;45(11):2444-55. doi: 10.1016/j.biocel.2013.07.019.

34. Lynch GS, Ryall JG. Role of beta-adrenoceptor signaling in skeletal muscle: implications for muscle wasting and disease. Physiol Rev. 2008 Apr;88(2):729-67. doi: 10.1152/physrev.00028.2007.

35. Whitaker RM, Corum D, Beeson CC, Schnellmann RG. Mitochondrial Biogenesis as a Pharmacological Target: A New Approach to Acute and Chronic Diseases. Annu Rev Pharmacol Toxicol. 2016;56:229-49. doi: 10.1146/annurev-pharmtox-010715-103155.

36. Yang $Y$, Creer A, Jemiolo B, Trappe $S$. Time course of myogenic and metabolic gene expression in response to acute exercise in human skeletal muscle. J Appl Physiol (1985). 2005 May;98(5):174552. doi: 10.1152/japplphysiol.01185.2004.

37. Brown JL, Rosa-Caldwell ME, Lee DE, Brown LA, Perry RA, Shimkus KL, Blackwell TA, Fluckey JD, Carson JA, Dridi S, Washington TA, Greene NP. PGC-1a4 gene expression is suppressed by the IL-6MEK-ERK 1/2 MAPK signaling axis and altered by resistance exercise, obesity and muscle injury. Acta Physiol (Oxf). 2017 Jun;220(2):275-288. doi: 10.1111/apha.12826.

38. Fortini P, lorio E, Dogliotti E, Isidoro C. Coordinated Metabolic Changes and Modulation of Autophagy during Myogenesis. Front Physiol. 2016 Jun 16;7:237. doi: 10.3389/fphys.2016.00237.

39. Barbosa MC, Grosso RA, Fader CM. Hallmarks of Aging: An Autophagic Perspective. Front Endocrinol (Lausanne). 2019 Jan 9;9:790. doi: 10.3389/fendo.2018.00790.

40. Escobar KA, Cole NH, Mermier CM, VanDusseldorp TA. Autophagy and aging: Maintaining the proteome through exercise and caloric restriction. Aging Cell. 2019 Feb;18(1):e12876. doi: 
10.1111/acel.12876.

41. Nyberg M, Mortensen SP, Cabo H, Gomez-Cabrera MC, Viña J, Hellsten Y. Roles of sedentary aging and lifelong physical activity in exchange of glutathione across exercising human skeletal muscle. Free Radic Biol Med. 2014 Aug;73:166-73. doi: 10.1016/j.freeradbiomed.2014.05.008.

42. Mullur R, Liu YY, Brent GA. Thyroid hormone regulation of metabolism. Physiol Rev. 2014 Apr;94(2):355-82. doi: 10.1152/physrev.00030.2013. PMID: 24692351; PMCID: PMC4044302.

43. Bloise FF, Cordeiro A, Ortiga-Carvalho TM. Role of thyroid hormone in skeletal muscle physiology. $\mathrm{J}$ Endocrinol. 2018 Jan;236(1):R57-R68. doi: 10.1530/JOE-16-0611.

\section{Table}

Table 1. qPCR primer sequences 


\section{Target gene}

AMP-activated protein kinase (AMPK)

Autophagy related 5 (ATG5)

Beta 2 adrenergic receptor (B2AR)

Deiodinase 2 (DIO2)

Deiodinase 3 (DIO3)

Estrogen related receptor alpha (ERRa)

Forkhead box 03 (FOXO3)

Glucose transporter 4 (GLUT4)

Glutathione synthetase (GSS)

Myocyte enhancer factor 2 (MEF2c)

Mechanistic target of rapamycin (mTOR)

Myogenic factor 5 (MYF5)

Myogenic differentiation 1 (MYOD)

Myogenin (MYOG)

Nuclear respiratory factor 2 (NRF2)

Nuclear respiratory factor 1 (NRF1)

Peroxisome proliferatoractivated receptor gamma coativator1-alpha (PGC-1a)

Peroxisome proliferatoractivated receptor gamma coativator1-beta (PGC-1 $\beta$ )

PGC-1a isoform 4 (PGC-1a4)

Ribosomal protein S13 (RPS13)

Sarco/endoplasmic reticulum Ca2+ ATPase isoform 1 (SERCA1)
Forward primer $\left(5^{\prime}\right.$ to $\left.3^{\prime}\right)$

TGTCCAGGGCTTGTTCTATTC

CCATTCCTTCCAAGCTAGTG

CCTGCTGACCAAGAATAAGG

GGCCCAAGTCATTCCTAATC

CCTCAAAGCAGCACCTAAA

GCTCCTCСTCTCATCATTTG

CCACCCTTGGCCTCTAAATAA

CTG GGCTTCTTCATCTTCAC

GTA AGCTGCTCTGAGGTAAAG

GCTGAAGAAGGAGATTTGTTTG

GGACTACAGGGAGAAGAAGA

CATCAGAGTCAAGTGGTCATAG

GCTTCTAGTTCCAGGCTTATC

GCCTTCTTCTTCCTGTGTATTA

CACAACGGACGACTTCTATG

CCCTGAATTGAGAGAGAAGAAG

GTGAGAACACACCAGAGAAAG

TCAACAACAGGGAGGTTAATG

GATGGCACTGTCTCACTTATC

GTCATCTCACCTCCCTGTAA

тСТСТСТСТСТСТСТСТСТст

CATGGGTGTCAGGATTAAGG

GCTCCAAGTGTGTGTCTATC

CACAGTCATTTCGCCTCT T

CAACCTTGTCCCTGGTTTAT

GCATCTTGAGAGGAACAGAAA

GTTCATCCGCTACCTCATTT

GTCACCAAGTTCACCCATAG
ATTCTCACTTGCCATCATTCT

AGGACTCGCTTGGTCTTAT 
$\mathrm{Ca} 2+$ ATPase isoform 2a

(SERCA2)

Superoxide dismutase 2 (SOD2)

TTTCTTTCTCCTGCCTGATG

ССТСТСТСАССАСТССТАТТ

Transcription factor $A$,

mitochondrial (TFAM)

CTCAAACTCCTGGCATCAA

GGTGGCTCACACCTATAATC

Thyroid receptor alpha (THRa) GTCACTCTCTGCCTTTAACC

CTCCTGACTCTTCTCGATCT

\section{Figures}

a

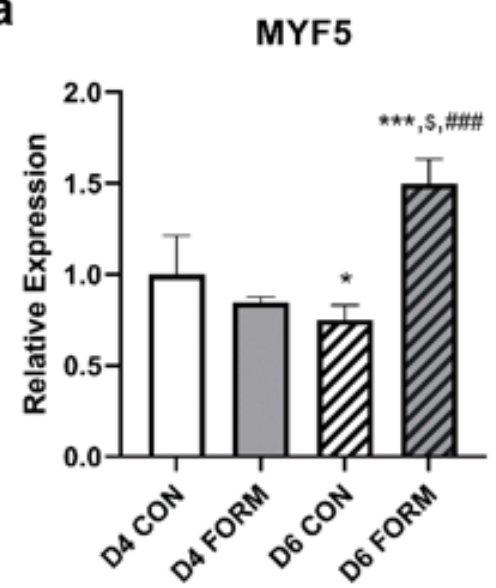

d

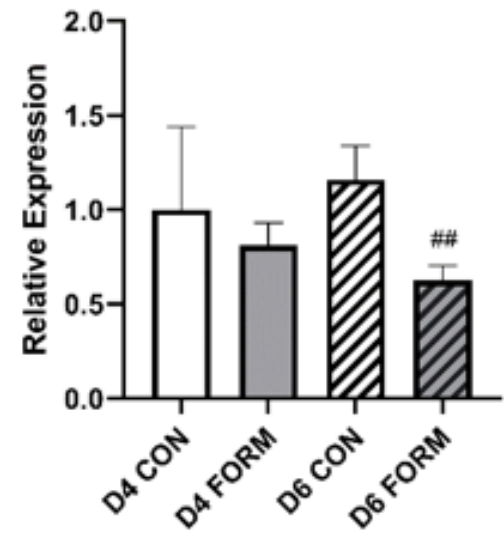

b

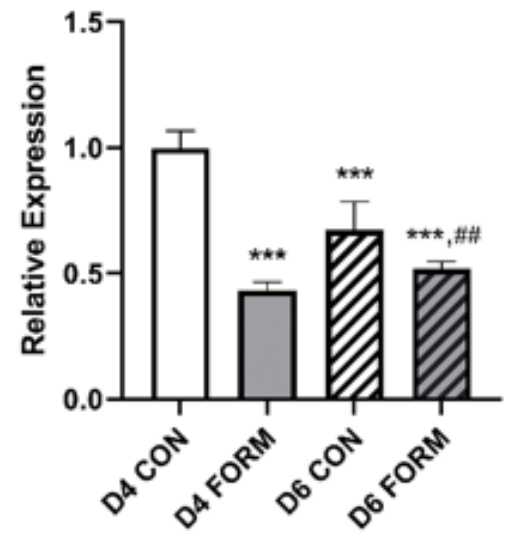

e

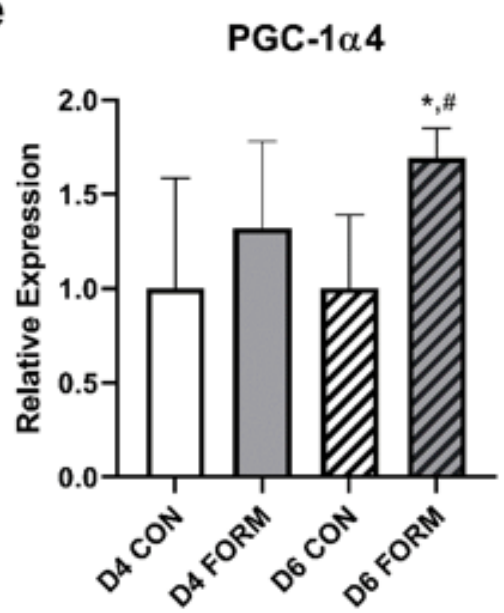

C

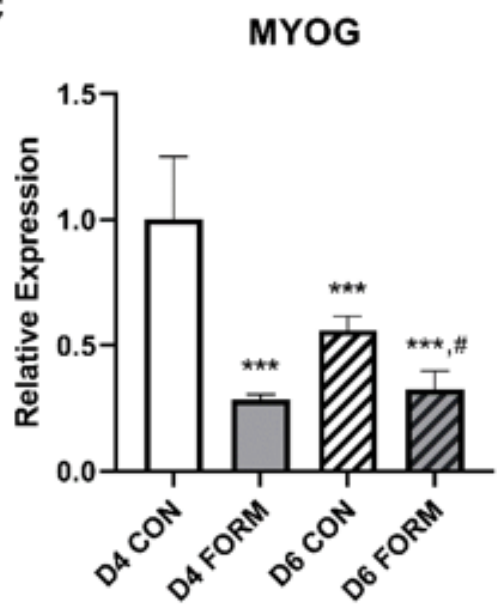

f

mTOR

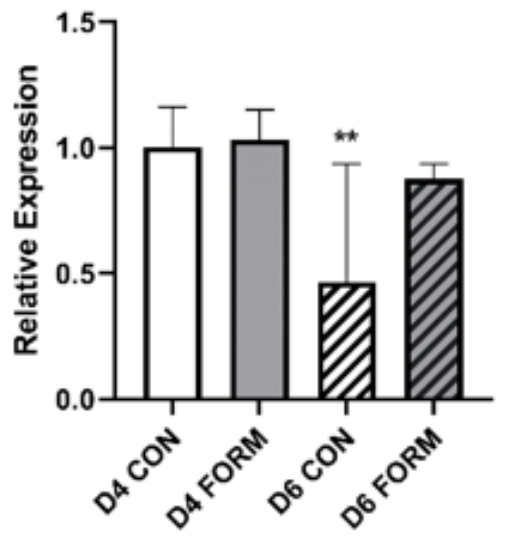

Figure 1

Formoterol influences SKM gene expression related to myogenesis. The expression of each gene (a-f) was normalized to the endogenous control RPS13 and expressed relative to D4 CON. $n=6$ per group. Significantly different compared to D4 CON: ${ }^{*} P<0.05,{ }^{\star} \mathrm{P}<0.01,{ }^{\star *} \mathrm{P}<0.001$. Significantly different 
compared to D4 FORM $\$ P<0.001$. Significantly different compared to D6 CON \#P $<0.05$, \#\#P<0.01, $\# \# \# P<0.001$. Data are presented as mean \pm SEM. One-way ANOVA with Tukey HSD post-test.

a

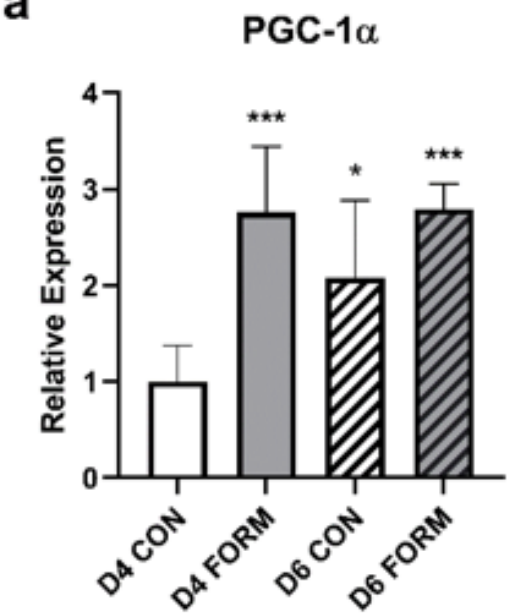

d

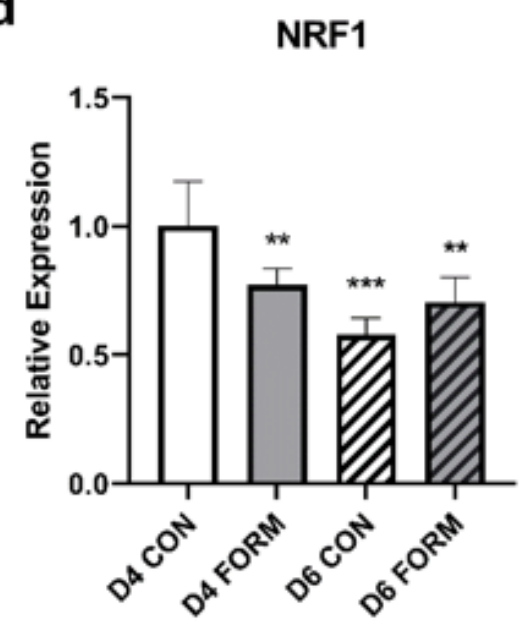

b

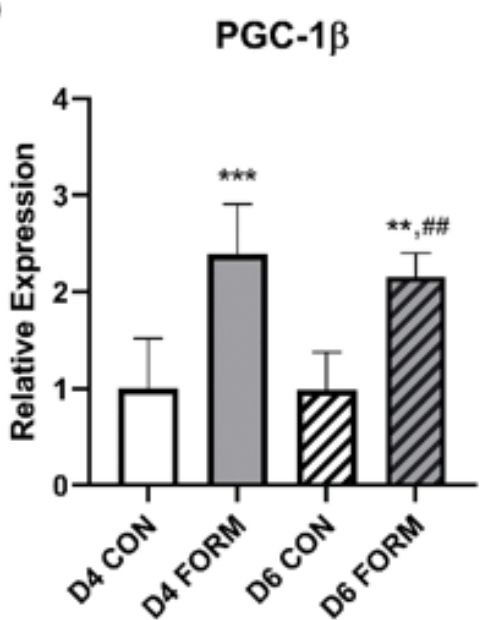

e

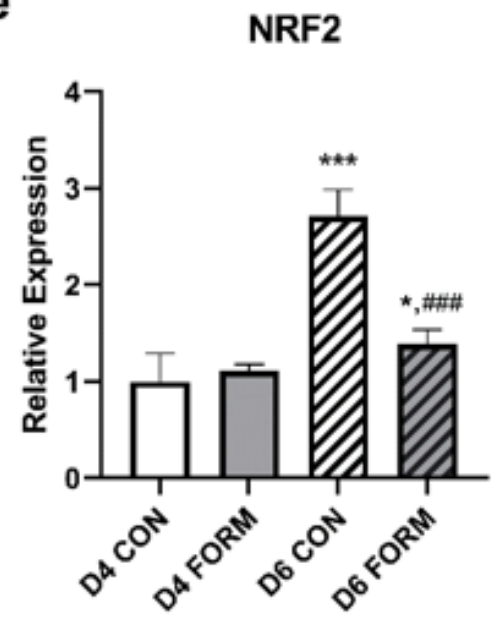

C

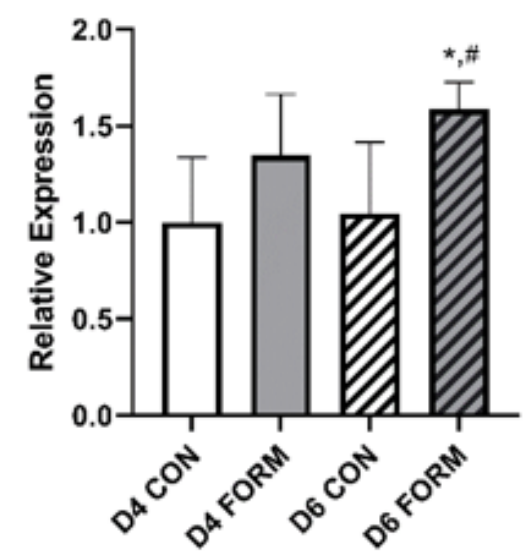

$\mathbf{f}$

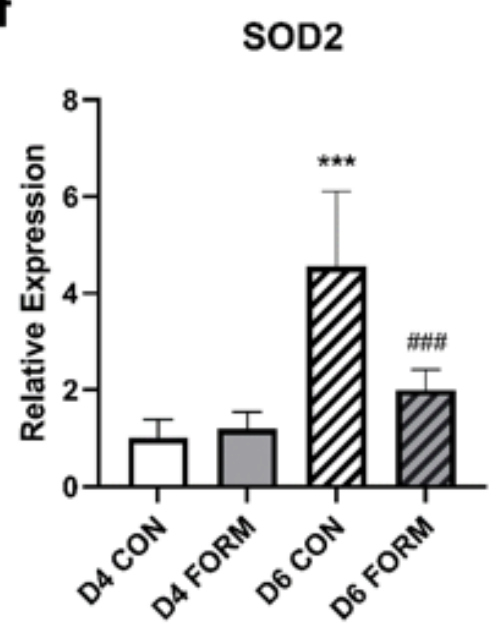

Figure 2

Formoterol stimulates expression of genes related to SKM mitochondrial biogenesis. The expression of each gene (a-f) was normalized to the endogenous control RPS13 and expressed relative to D4 CON. $\mathrm{n}=$ 6 per group. Significantly different compared to D4 CON: ${ }^{*}<<0.05$, ${ }^{\star *} P<0.01$, ${ }^{\star *} P<0.001$. Significantly different compared to D6 CON \#P $<0.05$, \#\#P $<0.01$, \#\#\#P $<0.001$. Data are presented as mean $\pm \mathrm{SEM}$. One-way ANOVA with Tukey HSD post-test. 
a

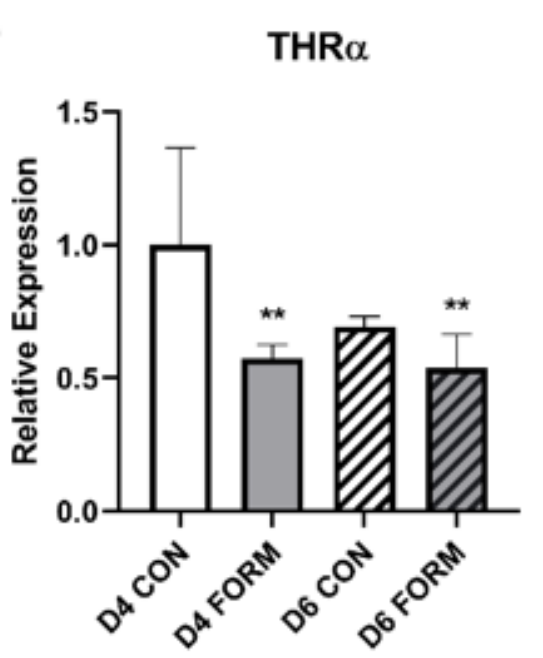

d

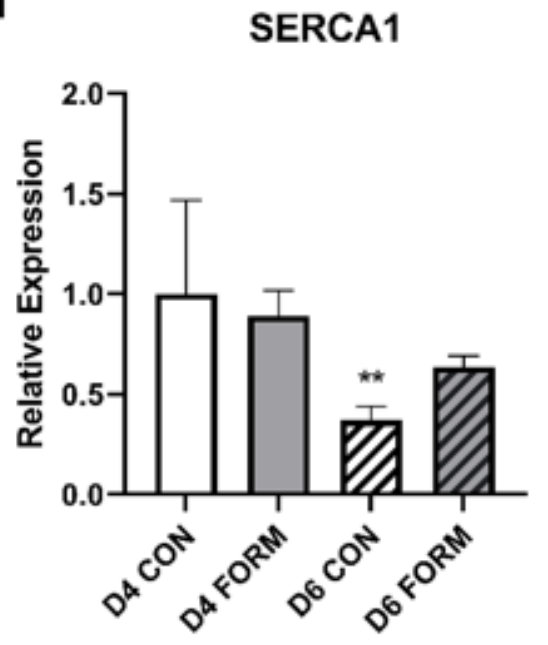

b

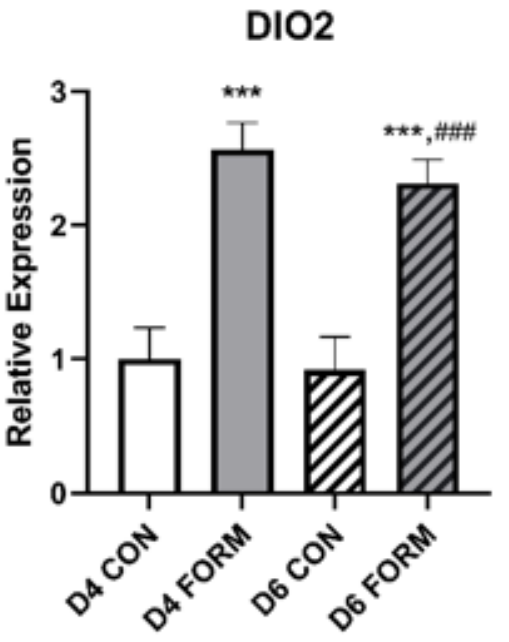

$\mathbf{e}$

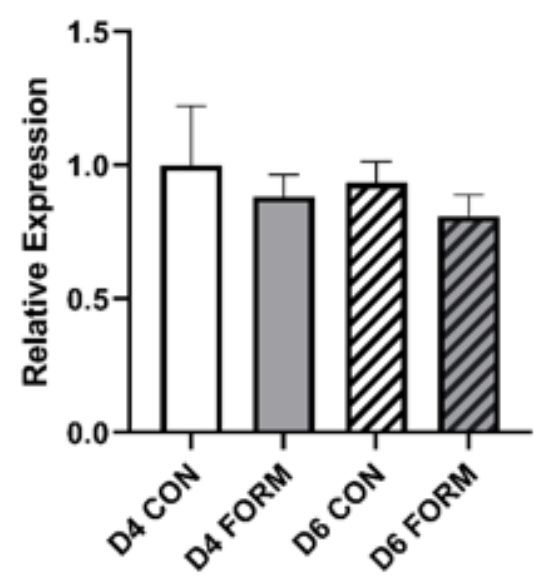

C

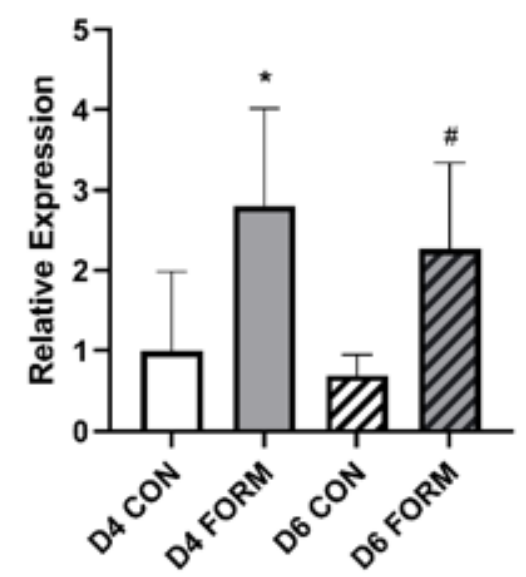

f

ERR $\alpha$

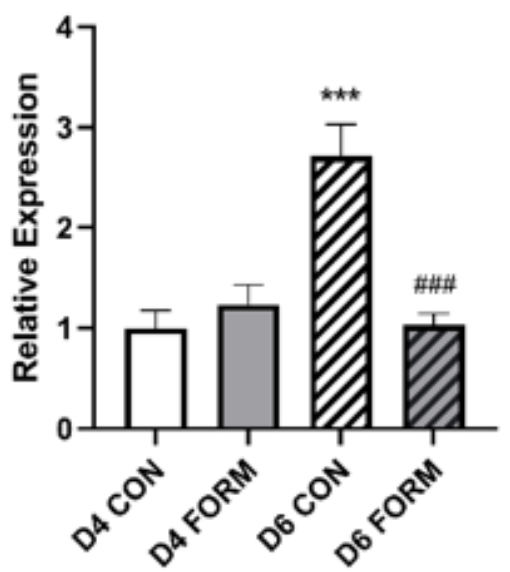

Figure 3

Formoterol affects intracellular SKM activity of genes related to thyroid hormone metabolism. The expression of each gene (a-f) was normalized to the endogenous control RPS13 and expressed relative to D4 CON. $n=6$ per group. Significantly different compared to D4 CON: ${ }^{*}<<0.05,{ }^{\star} * P<0.01,{ }^{\star \star} P<0.001$. Significantly different compared to D6 CON \#P $<0.05$, \#\#\#P $<0.001$. Data are presented as mean \pm SEM . One-way ANOVA with Tukey HSD post-test. 
a

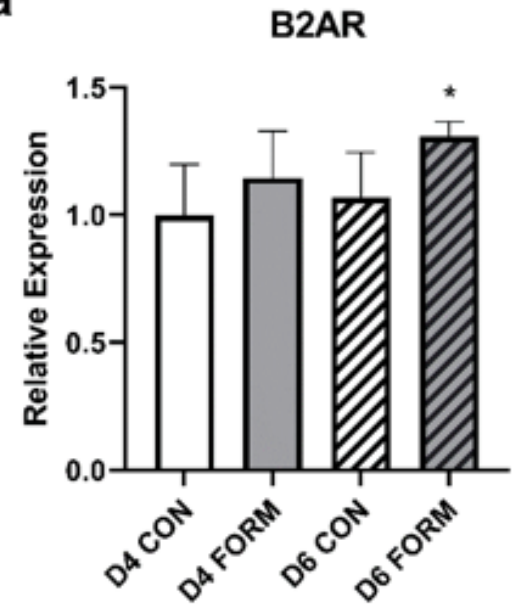

d

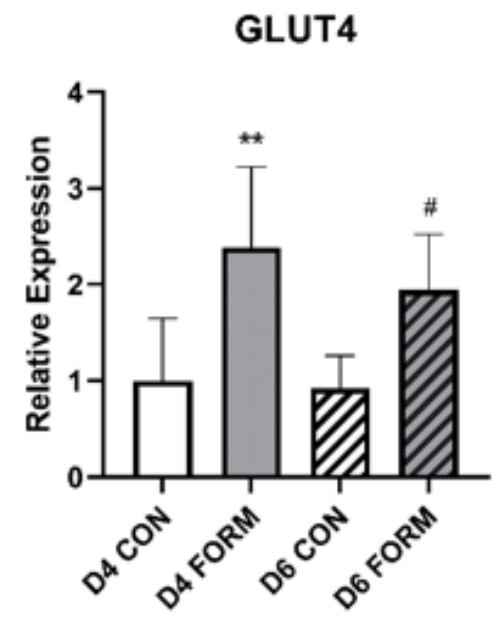

b

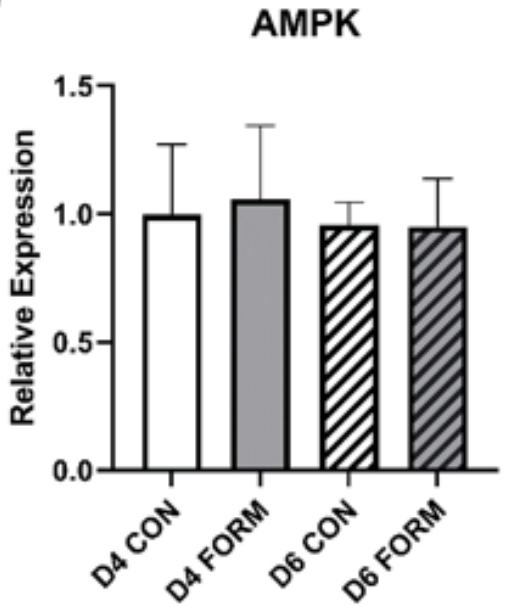

e

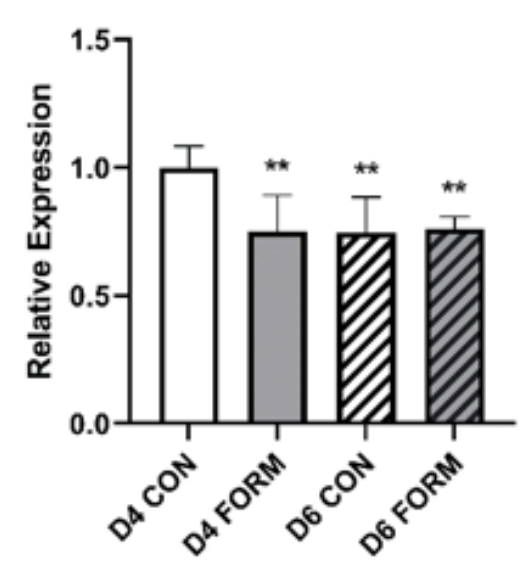

C

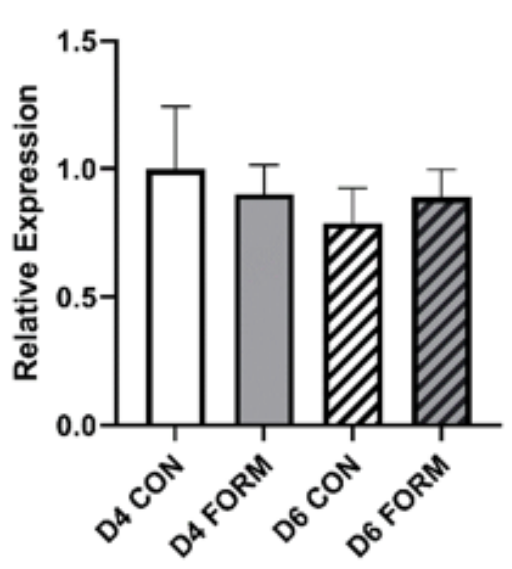

$\mathbf{f}$

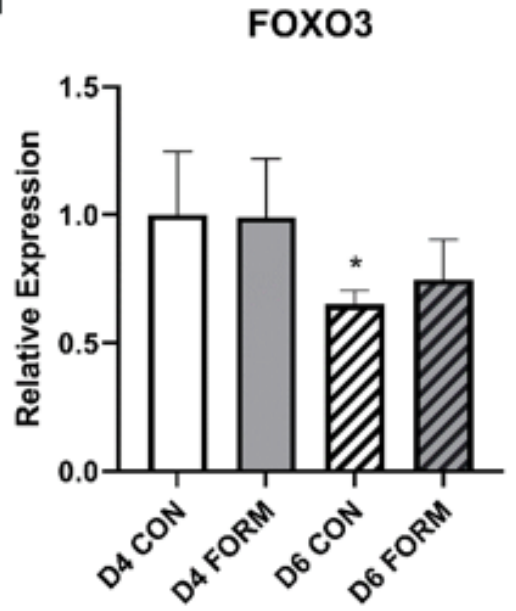

Figure 4

Formoterol increases SKM $\beta 2$-adrenergic receptor gene expression and influences genes related to cellular homeostasis. The expression of each gene (a-f) was normalized to the endogenous control ribosomal protein S13 (RPS13) and expressed relative to D4 CON. n = 6 per group. Significantly different compared to D4 CON: ${ }^{P}<0.05, \star \star P<0.01$. Significantly different compared to D6 CON \#P $<0.05$. Data are presented as mean \pm SEM. One-way ANOVA with Tukey HSD post-test. 